\title{
Heat Transfer Predictions for Carbon Dioxide in Boiling Through Fundamental Modelling Implementing a Combination of Nusselt Number Correlations
}

\author{
L. Makaum, P.v.Z. Venter and M. van Eldik
}

\begin{abstract}
Refrigerants with a high global warming potential are being phased out. The result is the utilisation of natural occurring gasses. Carbon dioxide $\left(\mathrm{CO}_{2}\right)$ possesses excellent heat transfer properties and is deemed as a viable heat pump refrigerant option. However, as of yet, it is unclear which Nusselt number correlations should be used to accurately predict convective heat transfer for $\mathrm{CO}_{2}$ in boiling.

This paper demonstrates how the combined utilisation of existing Nusselt number correlations can be used to accurately predict the total heat transfer of a $\mathrm{CO}_{2}$-to-water evaporator. The well-known correlations of Dittus-Boelter together with Forster and Zuber are used in a fundamentally based simulation model. For water, saturatedliquid and superheated $\mathrm{CO}_{2}$ vapour the Dittus-Boelter correlation is applied, whereas Forster and Zuber's correlation is incorporated for the boiling vapour. This model proves to be accurate within $3.2 \%$ when compared to our experimental $\mathrm{CO}_{2}$ heat pump test bench.
\end{abstract}

Keywords $-\mathrm{CO}_{2}$; two-phase; boiling heat transfer; evaporator; correlation.

\section{INTRODUCTION (HEADING 1)}

A common attempt to combat global warming is the reduction of greenhouse gas emissions. Carbon dioxide $\left(\mathrm{CO}_{2}\right)$ is typically viewed as the main contributor to global warming and numerous studies place emphasis on reducing $\mathrm{CO}_{2}$ emissions. It is furthermore used as a comparative measurement for greenhouse gasses' potential contribution towards global warming. The global warming potential (GWP) is a relative measure of the heat trapping effect of a gas in comparison to an equal mass of $\mathrm{CO}_{2}$ over a given quantity of time in the atmosphere [1].

One of the most commonly known refrigerants used after the 1987 Montreal Protocol banned CFC's and HCFC's, has been $\mathrm{R}-134 \mathrm{a}$. However, for $\mathrm{R}-134 \mathrm{a}$ the $\mathrm{GWP}=1300$. This and the typically high GWP of the mainstream refrigerants utilised in refrigeration cycles led researchers to explore natural occurring

P.v.Z. Venter is with the School of Mechanical and Nuclear Engineering, North-West University. Potchefstroom, South-Africa.

L. Makaum was with School of Mechanical and Nuclear Engineering, North-West University. Potchefstroom, South-Africa (12330825@nwu.ac.za).

M. van Eldik, School of Mechanical and Nuclear Engineering, North-West University. Potchefstroom, South-Africa. gasses as viable replacements. Carbon dioxide is such an option [11].

Heat pump cycles are widely used to transfer thermal energy to a secondary medium, typically water. Great care needs to be taken in the design and manufacturing of such a cycle. Not only should the heat transfer rates be cautiously calculated, but also the physical sizing of the components accurately determined. One of these components is the evaporator. An evaporator is a heat exchanger and it is critical to compute its dimensions in order to withdraw sufficient energy from the environment.

The correct sizing of an evaporator depends on the precision of the simulation model. The accuracy of such a model depends on the calculations of the numerically based Nusselt number correlations to predict the convection heat transfer coefficients. According to [11] there exists a number of Nusselt number correlations for $\mathrm{CO}_{2}$, but due to limited available data it is unsure as to what correlation(s) should be used. This paper will demonstrate, through a detailed evaporator simulation model, an accurate Nusselt number correlation to be used to for $\mathrm{CO}_{2}$ in boiling.

\section{LITERATURE STUDY}

\section{A. Rationale Behind the use of $\mathrm{CO}_{2}$}

Properties such as non-flammability, non-toxicity, low price, availability and no need for recycling, are just some of the benefits that carbon dioxide exhibits. It also offers great heat transfer characteristics due to low viscosity and high thermal conductivity. [10]

Low critical temperatures and high working pressures are required to use $\mathrm{CO}_{2}$ as a refrigerant under typical heat pump conditions. Carbon dioxide has a considerable low critical point (7.38 $\mathrm{MPa}$ and $31.1^{\circ} \mathrm{C}$ ) compared to other refrigerants. In a conventional heat pump cycle, low temperatures have disadvantages due to the limitation on the range of the operating temperatures. The enthalpy of vaporization reduces at temperatures just below the critical point which leads to a reduction in the heating capacity and poor system performance. Thus, it is important to avoid operation near heat rejection temperatures (critical point) [1].

Comparing the properties of carbon dioxide with R22 and $\mathrm{NH}_{3}$, the volumetric refrigerating capacity of carbon dioxide is 7 and 8 times greater than that of $\mathrm{R} 22$ and $\mathrm{NH}_{3}$ respectively, at a 
temperature of $-40^{\circ} \mathrm{C}$. This reduces the cross-sectional area required in a carbon dioxide plant by an eighth in comparison to what is needed in a $\mathrm{NH}_{3}$ plant. In other words, smaller cross-sections and compressors can be used which leads to lower charges and more economical plants. Therefore, the high pressure and density leads to high temperature heat transfer during evaporation. This property is the only one that makes carbon dioxide compatible with normal evaporator geometries [5].

\section{B. Previous Studies on Evaporative Heat Transfer}

An investigation on the heat transfer coefficient was conducted by Chen in 1966. He developed a flow boiling correlation which was divided into two different coefficients for two-phase flow. The two divisions were called the nucleate pool boiling coefficient and the bulk convective coefficient [2].

The study of [7] investigated the two-phase characteristics in a smooth, horizontal, stainless steel tube. A large variety of values were obtained for the local heat transfer.coefficient.and.the.local.pressure.gradient.in a $6 \mathrm{~mm}$ inner diameter tube. Mass flux, heat flux, saturation temperature and vapour quality were the important factors during the corresponding tests for uncontaminated $\mathrm{CO}_{2}$. The operating conditions varied for the saturated pressure from $28.2 \mathrm{bar}-$ 40.4bar, for saturated temperature from $-7.8^{\circ} \mathrm{C}-5.8^{\circ} \mathrm{C}$ and heat fluxes from $10.1 \mathrm{~kW} / \mathrm{m}^{2}-20.3 \mathrm{~kW} / \mathrm{m}^{2}$. The experimental data was evaluated according to the single-sample analysis suggested by [8]. The measurement of uncertainty ranged between $3.4 \%$ and $6.5 \%$ for the local heat transfer coefficient and between $0.2 \%$ and $2.4 \%$ for the local pressure gradient. Conclusions were drawn on whether the heat transfer coefficient and pressure gradient were dependant on the vapour quality, saturation temperature, mass flux and heat flux or not. The heat flux had a remarkable influence on the heat transfer coefficient for all the observed vapour qualities. The pressure gradients were strongly influenced by the increase of saturation temperature due to the increase in vapour density and decrease in mean velocity [7].

\section{THEORY}

\section{A.Principle of a Heat Exhanger}

Heat can be defined as the energy that passes spontaneously between a system and its surroundings. Heat flows naturally from a warm to a cooler medium. An increase in the temperature difference between the bodies leads to an increase in heat transfer.

Any conventional evaporator can be used for a supercritical system with $\mathrm{CO}_{2}$ as the working fluid. The heat causing the refrigerant to boil is the heat absorbed from the environment (the water in the annulus).

There are numerous configurations being used for heat exchange like parallel flow, counter flow and cross flow. In the counter flow configuration, the hot and cold fluids enter at opposite sides of the evaporator and travels to the opposite end. The fluids still flow parallel but in opposite directions which provides a uniform temperature difference.

\section{B. Relevant Theory to Perform an Evaporator Simulation}

The evaporator from the experimental test bench has a tube-in-tube heat exchanger as shown in Fig. 1. Water flows in the outer annulus and $\mathrm{CO}_{2}$ on the inside. The following theory can be found in [6] and [9]. The cross-sectional area of a tube is calculated by:

$$
A={ }^{1} / 4 \cdot \pi \cdot D_{i, i}{ }^{2}
$$

While the concentric cross-sectional area must be calculated by (2) at the inner and outer diameter.

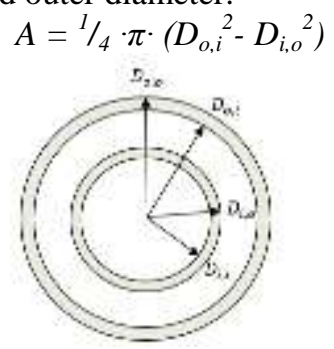

Fig. 1: Cross-sectional area of concentric tubes

The flow area is the surface area of the tube in contact with the fluid and can be calculated by the outer surface equations:

$$
A_{\text {outside }}=\pi \cdot D \cdot L
$$

The hydraulic diameter of a pipe is equivalent to diameter of a circle inscribed within the wetted perimeter. For a simple tube the hydraulic diameter is the diameter of the pipe, where for flow in the annulus the hydraulic diameter is:

$$
D_{h}=D_{o, i}-D_{i, o}
$$

\section{Conduction}

Conduction heat transfer is the transfer of energy through a solid medium. We are only interested in the conduction through the tube wall from $T_{s, 1}$ to $T_{s, 2}$. This can be expressed by Fourier's Law in terms of cylindrical coordinates:

Where:

$$
\begin{aligned}
& q_{\text {rcond }}=-k \cdot A \cdot{ }^{d T} / d r \text { where } \\
& q_{r c o n d}=2 \cdot \pi \cdot L \cdot k \cdot\left(T_{s, 1}-T_{s, 2}\right) \div \ln \left(r_{2} / r_{l}\right)
\end{aligned}
$$

$q_{\text {rcond }}=$ Heat transfer rate for conduction [W]

$L=$ Length of the tube [m]

$k=$ Thermal conductivity of a material $[\mathrm{W} / \mathrm{m} \cdot \mathrm{K}]$

$T_{s}=$ Temperature of the surface $[\mathrm{K}]$

$T=$ Temperature in $[\mathrm{K}]$ where

$T_{s, 1}$ the hot fluid temperature on the inside of the tube $>T_{s, 2}$ the cold fluid temperature on the outside of the tube.

$r=$ Radius [m] where $r_{1}$ represent the inner wall radius and $r_{2}$ the outer wall radius.

This will be used to calculate the conduction through the wall of the inner stainless steel tube. The outer tube is well insulated and therefore the assumption is made that there is no heat loss to the surroundings.

\section{Convection}

Convection heat transfer is the transfer of energy from a surface to a moving fluid or the other way around.

We are interested in the convection from the fluid to the wall and from the wall to the fluid. Thus, from $T_{\infty, 1}$ to $T_{s, 1}$ and then from $T_{s, 2}$ to $T_{\infty, 2}$. This can be expressed by:

$$
\begin{aligned}
& \mathrm{q}_{\text {rconv }}=\mathrm{h} \cdot \mathrm{A} \cdot\left(T_{s}-T_{\infty}\right) \\
& \mathrm{q}_{\text {rconv }}=\mathrm{UA} \cdot\left(T_{s}-T_{\infty}\right) \\
& U A=1 / R_{\text {convection }}
\end{aligned}
$$




$$
\begin{array}{r}
R_{\text {convection }}=1 /(2 \cdot h \cdot \pi \cdot r \cdot L) \\
\mathrm{q}_{\text {rconv }}=(2 \cdot h \cdot \pi \cdot r \cdot L) \cdot\left(T_{s}-T_{\infty}\right)
\end{array}
$$

These equations will be used to calculate the convection from the hot fluid to the surface of the tube and then from the surface of the tube to the cold fluid.

Where:

$q_{\text {rconv }}=$ Heat transfer rate for convection [W]

$L=$ Length of the tube [m]

$h=$ Convection heat transfer coefficient for the fluid $\left[\mathrm{W} / \mathrm{m}^{2} \cdot \mathrm{K}\right]$

$T_{\infty}=$ Temperature of the fluid $[\mathrm{K}]$

$r=$ Radius [m] where $r_{1}$ represent the inner wall radius and $r_{2}$ the outer wall radius

\section{E. Reynolds Number and Mass Flow}

The Reynolds number ( $\mathrm{Re})$ is defined as the ratio of inertial resistance to viscous resistance for a flowing fluid.

$$
R e=\rho V l / \mu=\rho U_{m} \mathrm{D} / \mu
$$

Where:

$R e=$ Reynolds number

$\rho=$ Density of the fluid $\left[\mathrm{kg} / \mathrm{m}^{3}\right]$

$V=$ Relative speed of fluid $[\mathrm{m} / \mathrm{s}]$

$l=$ Length of the system $[\mathrm{m}]$

$\mu=$ Dynamic viscosity of the fluid $\left[\mathrm{Ns} / \mathrm{m}^{2}\right]$

$U_{m}=$ Mean fluid velocity over cross section $[\mathrm{m} / \mathrm{s}]$

$\mathrm{D}=$ Tube diameter

For internal flow the critical Reynolds number is $R e_{\text {crit }}=$ 2300. If $R e_{D}$ is smaller or equal to $R e_{c r i t}$, the flow is laminar and when $R e_{D}$ is greater than 2300 , the flow is turbulent. It is important to consider the type of flow that is present in the pipe because it will determine which Nusselt correlation to use to determine the heat transfer coefficient.

The mass flow $[\mathrm{kg} / \mathrm{s}$ ] for a fluid is a function of the speed of the fluid, the density and the cross-sectional area of the tube:

$$
\dot{m}=\rho \cdot V \cdot A
$$

\section{F. Nusselt Numbers}

Different geometry of a heat exchanger requires different correlations to obtain the heat transfer coefficient. For this study, we will look at correlations for counter flow in a tube in tube evaporator. Most correlation will take the "Nusselt form":

$$
N u=a \cdot \operatorname{Re}^{b} \cdot \operatorname{Prc}
$$

$$
N u=h_{c} \cdot D h / k
$$

Where:

$R e=$ Reynolds number

$\operatorname{Pr}=$ Prandlt number

$D_{h}=$ hydraulic diameter

$h_{c}=$ convective heat transfer coefficient

$k=$ thermal conductivity

$a, b, c=$ correction factors

Evaluate the fluid properties at the "bulk average" temperature, unless stated differently.

$$
\begin{gathered}
T_{b}=1 / 2 \cdot\left(T_{\text {in }}+T_{\text {out }}\right) \\
\Delta T_{\text {water }}=1 / 2 \cdot\left(T_{\text {water, } \text { in }}+T_{\text {water, out }}\right)-T_{\text {wall,water }} \\
\Delta T_{\mathrm{CO} 2}=T_{\text {wall,CO2 } 2}-1 / 2 \cdot\left(T_{\mathrm{CO} 2 \text {, }{ }_{\text {in }}}+T_{\mathrm{CO}, \text { out }}\right)
\end{gathered}
$$

\section{G. LMTD-Method and Heat Transfer}

The log mean temperature difference method is used when the inlet temperatures and mass flow rates are known and the outlet temperatures needs to be calculated. The heat transfer rate of the hot and the cold stream can be calculated by:

$$
\begin{aligned}
& Q=\dot{m}_{h} \cdot\left(h_{h, \text { out }}-h_{h, \text { in }}\right) \\
& Q=\dot{m}_{c} \cdot\left(h_{c, \text { out }}-h_{c, \text { in }}\right)
\end{aligned}
$$

With constant specific heats when no phase change occurs at steady state:

$$
\begin{aligned}
& Q=\dot{m}_{h} \cdot c_{p, h}\left(T_{h, \text { out }}-T_{h, \text { in }}\right) \\
& Q=\dot{m}_{c} \cdot c_{p, c}\left(T_{c, \text { out }}-T_{c, \text { in }}\right)
\end{aligned}
$$

Two other equation used to calculate the heat transfer is:

$$
\begin{aligned}
& Q=U A \cdot \Delta T_{l m} \\
& Q=h_{c} \cdot A_{\text {outside }} \cdot \Delta T
\end{aligned}
$$

Where $\Delta T_{l m}$ is, the log mean temperature difference:

$$
\Delta T_{l m}=\left(\Delta T_{2}-\Delta T_{1}\right) \div\left(\ln \Delta T 2 / \Delta T_{l}\right)
$$

And $\Delta T_{1}$ and $\Delta T_{2}$ for the counter flow configuration:

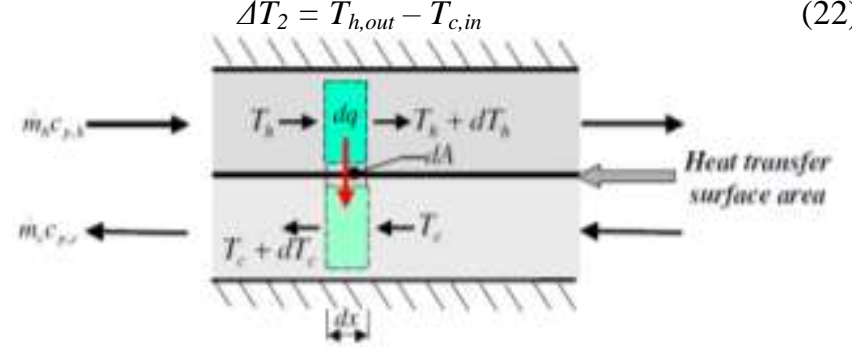

Fig. 2: Counter flow configuration

It is important to note that it is possible for the outlet temperature of the cold fluid to exceed the outlet temperature of the hot fluid. The temperature distribution for the counter flow configuration can be seen in Fig. 3 below.

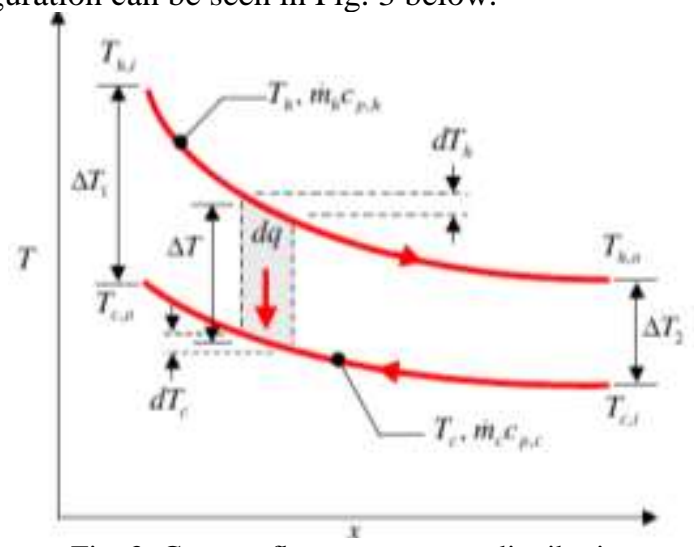

Fig. 3: Counter flow temperature distribution

\section{H. Correlation}

When considering a correlation or the use of several correlations, a few aspects should be taken into account:

1. What does the geometry look like? (Flow through a pipe, around an object, over a plane, etc.)

2. Is there any phase change in the substance? (Liquid to vapour or vapour to liquid)

3. What type of flow regime occurs? (Use the Reynolds number to predict laminar, transition, or turbulent flow.) 
4. Is natural convection important when the flow is laminar? (Use the Grashof number)

After a number of $\mathrm{Nu}$ correlations were investigates, the following combinations of equations and correlations were used to determine the convective heat transfer of two-phase $\mathrm{CO}_{2}$ in boiling. The existence of nucleate boiling depends on the local wall superheat defined as [3]:

$$
\left(\Delta T_{\text {sat }}\right)_{\text {wall }}=T_{\text {wall, }, \mathrm{CO} 2}-T_{\text {sat, } \mathrm{CO} 2}
$$

With $T_{\text {wall,CO2 }}$ the wall temperature of the inner tube and $T_{\text {sat,CO2 }}$ the saturation temperature at the corresponding pressure point. [3] correlation which was used to initiate nucleation states the following:

$$
\left(\Delta T_{\text {sat }}\right)_{\text {wall, } \text {,rit }}=\left(8 \cdot \sigma \cdot T_{\text {sat }} \cdot q^{\prime \prime}\right) \div\left(\rho_{g} \cdot h_{f g} \cdot k_{f}\right)
$$

Where $\sigma$ represent the surface tension.in $[\mathrm{N} / \mathrm{m}], T_{\text {sat }}$ the saturation temperature in $[\mathrm{K}]$, the vapour density $\rho_{\mathrm{g}}$ in $\left[\mathrm{kg} / \mathrm{m}^{3}\right]$, $h_{f g}$ the latent heat of vaporization in $[\mathrm{J} / \mathrm{kg}]$ and $k_{f}$ the thermal conductivity in $\left[\mathrm{W} / \mathrm{m}^{2} \mathrm{~K}\right]$.

$$
\begin{gathered}
N u=h_{c h t, f} D_{h} / k_{f} \\
\mathrm{Nu}=0.023\left(\dot{\mathrm{m}} \cdot \mathrm{D}_{\mathrm{h}}(1-\mathrm{x}) / \mathrm{A}_{\mathrm{ff}} \cdot \mu_{\mathrm{f}}\right)^{0.8} \cdot\left(\mu_{\mathrm{f}} \cdot \mathrm{C}_{\mathrm{pf}} / \mathrm{k}_{\mathrm{f}}\right)^{0.4}
\end{gathered}
$$

Where $x$ represents the quality [-] of the $\mathrm{CO}_{2}$. The Dittus Boelter equation is used for $\mathrm{CO}_{2}$ in the two-phase region.

The well-known Dittus-Boelter was used to calculate the single-phase liquid convective heat transfer coefficient $h_{\text {chtc,f }}$ between the wall and the carbon dioxide, assuming the carbon dioxide fills the whole tube. The combination of convective heat transfer and nucleate boiling of vertical plain tubes is stated in equation (26) [2].

$$
h_{\text {two }, \text { phase }}=\left(F \cdot h_{\text {cht }, f}+S \cdot h_{\text {bhtc, }, Z Z}\right) \cdot \Delta T_{\text {sat }}
$$

$h_{b h t, F Z}$ is the Forster and Zuber heat transfer coefficient for nucleate boiling [4].

$h_{\text {bhtc }, F Z}=\left(0.00122 \cdot\left(\Delta T_{\text {sat }}\right)^{0.24} \cdot\left(\Delta P_{\text {sat }}\right)^{0.75} \cdot c p_{f}^{0.45} \cdot \rho_{f}^{0.49} \cdot k_{f}^{0.79}\right) \div$ $\left(\sigma^{0.5} \cdot h_{f g}^{0.24} \cdot \mu_{f}^{0.29} \cdot \rho_{g}{ }^{0.24}\right)$

In (27), $\Delta P_{\text {sat }}$ is the difference in saturation pressure at the wall and bulk temperatures. In equation (26), F is the two-phase multiplier and $\mathrm{S}$ the suppression factor for nucleate boiling.

$$
F=2.35 \cdot\left(1 / x_{t t}+0.213\right)^{0.736}
$$

With $0.1<1 / x_{t t} \leq 100$ and $F=1.0$ if $1 / x_{t t} \leq 0.1$

$x_{t t}$ is the Lockhart-Martinelli parameter defined as:

Fig

$$
x_{t t}=\left({ }^{1} / \mathrm{x}-1\right)^{0.9} \cdot\left(\rho_{\mathrm{g}} / \rho_{\mathrm{f}}\right)^{0.5} \cdot\left(\mu_{\mathrm{f}} / \mu_{\mathrm{g}}\right)^{0.1}
$$

The correlation for calculating the suppression factor is given by:

$$
\begin{aligned}
& S=\left(1+2.53 \times 10^{-6} \operatorname{Re}_{t p}{ }^{1.17}\right)^{-1} \\
& \operatorname{Re}_{t p}=\operatorname{Re}_{f} \cdot F^{1.25}
\end{aligned}
$$

With $\operatorname{Re}_{\mathrm{f}}$, the liquid-base Reynolds number in the tube given by:

$$
R e_{f}=\left|\dot{m}_{f} \cdot D h \div \mu_{f} \cdot A f f\right|
$$

The Dittus-Boelter correlation will be used for the water flowing in the annulus of the evaporator, as well as the superheated $\mathrm{CO}_{2}$.

Where:

$$
N u_{D}=0.023 R e_{D}^{0.8} P^{\mathrm{n}}
$$

$R e_{D}=$ Reynolds number

$\operatorname{Pr}=$ Prandlt number

$\mathrm{n}=0.3$ for water in cooling and 0.4 for $\mathrm{CO}_{2}$ in heating.

\section{EXPERIMENTAL SETUP}

The test bench used for this paper is a full working heat pump cycle consisting of a compressor, gas-cooler, expansion valve and an evaporator. A number of steady state experiments were performed. For each test the mass flow rates of the $\mathrm{CO}_{2}$ and water was measured. Water temperature measurements along the $16 \mathrm{~m}$ long evaporator were taken for every two meters, as well as the $\mathrm{CO}_{2}$ 's inlet and outlet temperature. At every instance pressure was measured, that includes the pressure for the $\mathrm{CO}_{2}$. The mass flow rates and evaporator dimensions are given in Tables 1 and 2 respectively.

\section{RESULTS}

\section{A. Input Parameters}

The input parameters used for the simulation model was obtained from the steady state experimental data from the test bench. Predictions obtained from the simulation model will be compared to the experimental results from the test bench. The necessary thermodynamic parameters and geometry boundary parameters used in the simulation model are found in TABLE 1 and 2 respectively.

TABLE 1:

THE INPUT VALUES FOR THE SIMULATION OBTAINED FROM THE TEST BENCH

\begin{tabular}{|l|c|l|l|l|}
\hline \multirow{2}{*}{} & \multicolumn{4}{|c|}{ Input parameters } \\
\cline { 2 - 5 } & $\mathbf{T}\left[{ }^{\mathbf{0}} \mathbf{C}\right]$ & $\mathbf{P}[\mathbf{k P a}](\mathbf{a b s})$ & $\mathbf{m}[\mathbf{k g} / \mathbf{s}]$ & $\mathbf{x}[-]$ \\
\hline Water & 19.5 & 350 & 0.17 & - \\
\hline $\mathrm{CO}_{2}$ & & 2890 & 0.35 & 1 \\
\hline
\end{tabular}

TABLE 2:

THE GEOMETRY OF THE TUBE IN TUBE EVAPORATOR

\begin{tabular}{|l|c|c|c|c|c|}
\hline \multirow{2}{*}{} & \multicolumn{5}{|c|}{ Input Geometry } \\
\cline { 2 - 6 } & $\boldsymbol{D}_{i, i}$ & $\boldsymbol{D}_{\boldsymbol{i}, \boldsymbol{o}}$ & $\boldsymbol{D}_{\boldsymbol{o}, \boldsymbol{i}}$ & $\boldsymbol{D}_{\boldsymbol{o}, \boldsymbol{o}}$ & $\boldsymbol{L}$ \\
\hline $\begin{array}{l}\text { Diameter } \\
{[\mathrm{mm}]}\end{array}$ & 15.8 & 21.3 & 26.6 & 33.4 & \\
\hline Length [m] & & & & & 16 \\
\hline
\end{tabular}

\section{B. Outputs and Comparison}

The outlet temperatures predicted by the simulation and the experimental temperatures are tabulated in TABLE 3 . Comparing the results of the simulation and the experimental values, it can be seen that the simulation slightly over predicts the heat transfer. The water temperature of the simulation is $0.55^{\circ} \mathrm{C}$ lower than the experimental value, resulting in a $3.2 \%$ over prediction of the total rate of heat transfer. The total heat transfer for the simulation as well as the experimental set-up is given in Table 4.

TABLE 3:

OUTPUT TEMPERATURES OF THE SIMULATION VS. EXPERIMENTAL DATA AT LENGTH INCREMENTS

\begin{tabular}{|c|c|c|l|l|l|}
\hline \multicolumn{5}{|c|}{ Output parameters } \\
\hline \multicolumn{3}{|c|}{ Simulation values } & \multicolumn{3}{c|}{ Experimental values } \\
\hline $\begin{array}{l}\mathrm{CO}_{2} \\
{\left[{ }^{\circ} \mathrm{C}\right]}\end{array}$ & $\begin{array}{l}\text { Water } \\
{\left[{ }^{\circ} \mathrm{C}\right]}\end{array}$ & $\begin{array}{l}\text { Length } \\
\text { increments } \\
{[\mathrm{m}]}\end{array}$ & $\begin{array}{l}\mathrm{CO}_{2} \\
{\left[{ }^{\circ} \mathrm{C}\right]}\end{array}$ & $\begin{array}{l}\text { Water } \\
{\left[{ }^{\circ} \mathrm{C}\right]}\end{array}$ & $\begin{array}{l}\text { Length } \\
\text { increments } \\
{[\mathrm{m}]}\end{array}$ \\
\hline-5.84 & 19.50 & 0 & -3.5 & 19.50 & 0 \\
\hline-5.84 & 16.32 & 2 & -3.7 & 17.45 & 2 \\
\hline
\end{tabular}




\begin{tabular}{|c|c|c|l|l|l}
\hline \multicolumn{6}{c}{ Output parameters } \\
\hline-5.84 & 13.06 & 4 & -3.9 & 14.75 & 4 \\
\hline-5.84 & 10.5 & 6 & -4.1 & 12.65 & 6 \\
\hline-5.84 & 8.44 & 8 & -4.4 & 10.35 & 8 \\
\hline-5.84 & 5.54 & 12 & -4.9 & 6.65 & 12 \\
\hline 2.63 & 1.65 & 16 & -4.40 & 2.20 & 16 \\
\hline
\end{tabular}

The temperature distribution of the water and the over the length of the evaporator is in Fig. The water temperature of the simulation decreases over the length of the evaporator from $19.5^{\circ} \mathrm{C}$ to $1.65^{\circ} \mathrm{C}$. As for the the temperature remains constant and starts to increase when entering the gas phase from $-5.84^{\circ} \mathrm{C}$ to $2.63^{\circ} \mathrm{C}$.

TABLE 4:

HEAT TRANSFERRED IN THE EVAPORATOR

\begin{tabular}{|l|l|}
\hline \multicolumn{2}{|c|}{ Heat transferred $[\mathbf{k W}]$} \\
\hline Simulation & Test bench \\
\hline 26.19 & 25.37 \\
\hline
\end{tabular}

The temperature distribution of the water and the $\mathrm{CO}_{2}$ of the simulation can be seen in Fig. 4. The effect of the counter flow configuration can also be seen.

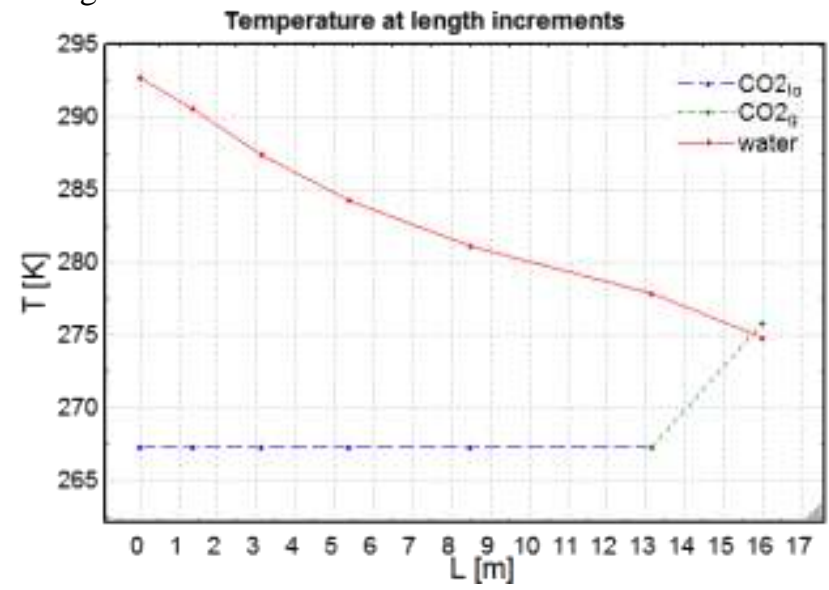

Fig. 4: Temperature distribution with respect to the simulation length increments

The enthalpy distributions over the length of the evaporator are plotted in Fig. 5 for the water and $\mathrm{CO}_{2}$ streams.

It is important to note that the $\mathrm{CO}_{2}$ undergoes a phase change at $13.16 \mathrm{~m}$ in the pipe. The first $13.16 \mathrm{~m}$ is referred to as the two-phase region while the excess $2.84 \mathrm{~m}$ is referred to as the gas region. During this gas region of the evaporator the $\mathrm{CO}_{2}$ is superheated by $8.47^{\circ} \mathrm{C}$ from $-5.48^{\circ} \mathrm{C}$ to $2.63^{\circ} \mathrm{C}$

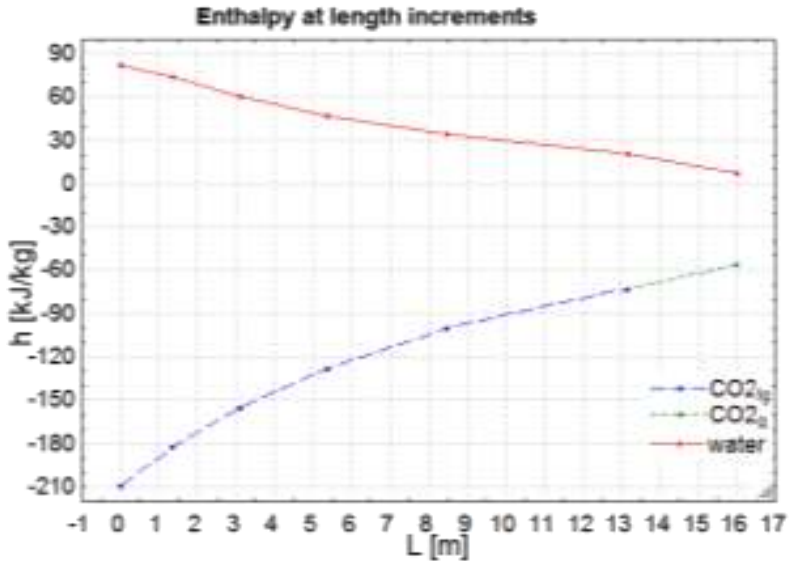

Fig. 5: Temperature distribution with respect to the simulation length increments

Fig6 illustrates the experimental water temperature at the experimental length increments versus the water temperature predicted by the simulation model over the length of the evaporator. For lower $\mathrm{CO}_{2}$ vapour qualities up till around $\mathrm{x}=0.8$ at $8 \mathrm{~m}$, the simulation model predicts higher convective heat transfer when compared with the experimental data. After a quality of 0.8 and for a gaseous $\mathrm{CO}_{2}$, the experimental data shows higher heat transfer than what was predicted, allowing for the total predicted heat transfer to move closer to the experimentally obtained values.

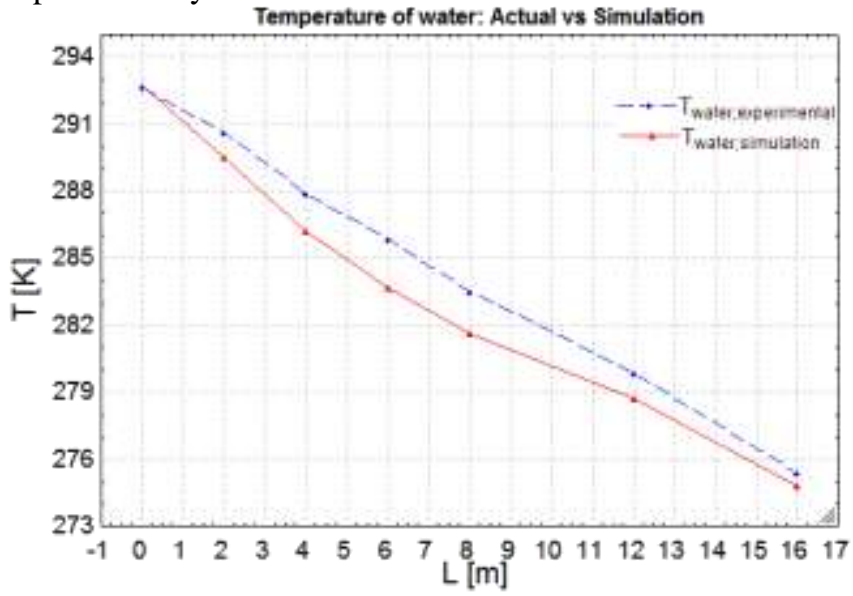

Fig 6: The experimental temperatures vs the simulation temperatures of the water at the respected length increments.

The total accumulated heat transfer over the length of the evaporator for the predicted vs. the experimental values can be seen in Fig. 7. 


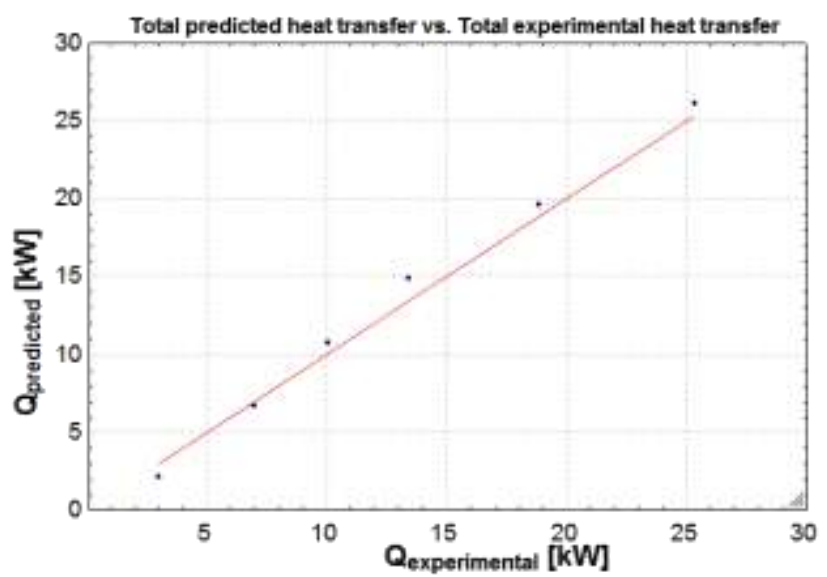

Fig. 7: Total heat transfer at experimental length increments

\section{CONCLUSION \& RECOMMENDATIONS}

This paper addressed the issue of accurately predicting the heat transfer of $\mathrm{CO}_{2}$ in boiling. A number of Nusselt $(\mathrm{Nu})$ correlations have been published over the last few years with no distinction as to what correlation or combination thereof performs the best. A simulation model was developed from fundamental principles. This model predicts the total heat transfer and therefore the outlet temperatures for a water-to- $\mathrm{CO}_{2}$ evaporator. A set of well-known convective heat transfer correlations was identified and utilised to predict the boiling two-phase heat transfer. It was found that by incorporating different, standard, Nu number correlations, that the total heat transfer over a $16 \mathrm{~m}$ evaporator was accurately predicted within $3.2 \%$ when compared with experimental results. The Dittus-Boelter Nu number correlation, published in 1923, was used for water and $\mathrm{CO}_{2}$ in the saturated liquid, as well as the superheated vapour regions. These were then combined with the Forster and Zuber Nu number correlation from 1955 for the saturated $\mathrm{CO} 2$ vapour. Results showed that up till around a vapour quality of 0.8 , the simulation model gradually over-predicted the heat transfer, whereas thereafter, it under-predicted. For the application of heat pump cycles this combination of $\mathrm{Nu}$ number correlations proved to be accurate when compared to the experimental data.

For further studies the accuracy of these correlation-combinations will be tested for various boiling temperatures. The temperature interval will lie within a typical $\mathrm{CO}_{2}$ heat pump cycle's operating boiling range. 